\title{
Publicidad exterior: Estudio exploratorio de recordación de marca y motivación de compra
}

\author{
Torres, Eduardo* \\ Muñoz, Juan Pablo**
}

\section{Resumen}

El presente estudio se centra en la medición del nivel de recordación de marca y del grado de motivación de compra del público consumidor que se expone a la publicidad exterior o en la vía pública (outdoor advertising). A través de una investigación exploratoria, por medio de entrevistas realizadas en diferentes lugares de la ciudad de Santiago de Chile y de sus estaciones del Metro (tren subterráneo), se pudo identificar, los diferentes factores que facilitan la recordación de marca, tales como el diseño, color y cobertura geográfica. Además, el estudio permitió conocer que un porcentaje importante de personas, se sienten motivadas a comprar a partir de la publicidad exterior, sin diferencias significativas según características demográficas como sexo y edad.

Palabras clave: Recordación de marca, motivación de compra, publicidad exterior.

Recibido: 06-08-31. Aceptado: 06-11-10

Doctor en Gestión, Universidad de Zaragoza (España). Académico e investigador, Facultad de Economía y Negocios, Universidad de Chile. Diagonal Paraguay 257, Santiago de Chile. E-mail: eduardot@negocios.uchile.cl Fono: +56 2 9783366. Fax: +56 22220639.

** Master of Business Administration, University of Chicago (EE.UU). Académico e Investigador, Facultad de Economía y Negocios. Universidad de Chile. E-mail: jpmunoz@negocios.uchile.cl Fono: +56 2 9783378. Fax: +56 22220639 . 


\title{
Outdoor Advertising: An Exploratory Research of Brand Recall and Purchase Motivation
}

\begin{abstract}
This study is focused on the analysis of brand recall and purchase motivation of consumers exposed to outdoor advertising. An exploratory research based on personal interviews conducted in different places and subway stations (Metro) in the city of Santiago de Chile, allowed for the identification of factors influencing brand recall in outdoor advertising, such as design, colors, localization and geographic coverage. The study also allowed us to state the hypothesis that an important percentage of consumers are motivated to purchase by outdoor advertising, with no significant differences in demographic characteristics, such as age and gender. At the end of the article, future research lines are suggested, in order to conclusively validate these results.
\end{abstract}

Key words: Brand recall, purchase motivation, outdoor advertising.

\section{Introducción}

La publicidad es uno de los elementos de la mezcla de marketing, que las empresas utilizan para lograr una diversidad de objetivos, como son introducir un nuevo producto en el mercado, posicionarse de acuerdo a un determinado concepto, aumentar la recordación de la marca o motivar la compra del público objetivo para lograrlo, las empresas no sólo recurren a los medios publicitarios tradicionales de uso masivo como son la televisión, la radio y la prensa; sino que se están complementando en forma creciente con otros medios menos tradicionales, entre los que se cuenta la publicidad exterior o en la vía pública. De hecho, la publicidad exterior ha experimentado un crecimiento importante en casi todo el mundo, debido a la creciente incorporación de nuevos soportes publicitarios y lugares donde exponerlos, como son por ejemplo, los automóviles particulares y el transporte público.
Frente a esta variedad de publicidad exterior, los directivos de marketing deben decidir en cuales de ellos enfatizar la inversión publicitaria, de acuerdo al nivel de recordación de marca que ellos generan. Desde esta perspectiva, la clave de la recordación se encuentra fundamentalmente en un conjunto de factores que deben ser gestionados a la hora de diseñar la pieza publicitaria. Entre estos factores se pueden destacar, los colores utilizados en el aviso, las características del texto, el tema en que se centra la publicidad y el lugar donde se expone el aviso.

Considerando lo importante que es para las empresas conocer el impacto que su publicidad exterior ha tenido sobre la recordación de las marcas y los factores que han influido en dicha recordación, el presente estudio se propone como objetivo, conocer las marcas publicitadas tanto en el Metro de Santiago como en la vía pública, que los consumidores recuerdan, así como las razones que contribu- 
yen a dicha recordación. Además, se propone identificar el grado de motivación de compra de estos productos publicitados y la relación que pudiera existir entre esta motivación y la edad y el sexo de las personas. Para lograr estos objetivos, se desarrollo de un estudio exploratorio, por medio de una encuesta aplicada a 300 personas en diferentes puntos de la ciudad de Santiago de Chile y de su transporte público subterráneo.

\section{Análisis de la publicidad exterior}

La publicidad exterior es una de las herramientas publicitarias más utilizadas por las marcas para darse a conocer, facilitar su recordación y motivar su compra. A pesar de que muchas veces la publicidad exterior pareciera no ser tomada en cuenta o ser considerada de menor categoría que la publicidad televisiva, en muy poco tiempo ha ido influyendo de manera creciente en la decisión de compra de los consumidores.

En la literatura especializada de marketing, la publicidad exterior (outdoor advertising) ha sido destacada por su cobertura, efectividad, espontaneidad y complejidad en su estructura y desarrollo. De hecho, la publicidad exterior ha sido descrita como uno de los medios de mayor alcance y uno de los más convenientes para llegar al público de manera masiva (Whitehill y Tinkham, 1990).

Además de su gran cobertura, la publicidad exterior se ha caracterizado por ser uno de los medios con los cuales se logra una sensibilidad casi inmediata en el consumidor. De hecho, ha sido considerado un medio propicio para reforzar la imagen de marca y generar un incentivo adicional para su recordación y motivación de compra. La publicidad exterior es más efectiva que otros medios publicitarios, debido a que el espectador es un receptor pasivo de información y por lo tanto es más permeable a recordar y aceptar el mensaje publicitario. En otros medios en cambio, el espectador es un procesador más activo de información y por lo tanto, puede evitar, ignorar o cuestionar el mensaje publicitario.

Por último, se debe tener presente, que a pesar de la sensibilidad, la publicidad exterior está condicionada por lo que en la literatura se denomina espontaneidad. La publicidad exterior, en mayor grado que los demás medios publicitarios, tiene la particularidad de ser un medio que a pesar de estar expuesto de manera constante, muchas veces no es visto o simplemente es ignorado por los consumidores (Young, 1984).

\subsection{Elementos claves de la publicidad exterior}

Desde hace varias décadas que en la literatura se pueden encontrar diferentes trabajos que han intentado conocer cuáles son los factores que intervienen en la efectividad de un aviso publicitario (Whitehill y Tinkham, 1990; Woodside, 1990; Bhargava y Donthu, 1993), en especial conocer cómo algunos factores concernientes a la propia estructura del aviso, es decir, su posición, color, número de palabras, entre otros. influyen en la recordación y en la motivación de compra hacia a la marca. Aunque los trabajos se han concentrado fundamentalmente en estos aspectos, los primeros estudios 
también se centraron en el consumidor, es decir, en el análisis de la actitud que éste tiene cuando se encuentra frente a un aviso y además de cómo esa actitud influye en su recordación. Este es el caso del estudio realizado por Hewett (1975). Este autor pudo determinar que cuando los consumidores tienen una actitud positiva frente al canal de comunicación (aviso) se produce una mayor recordación, a diferencia de cuando se tiene una actitud negativa, que es donde se produce un menor grado recordación por parte del consumidor.

En cuanto a los estudios que han analizado las características del aviso, podemos destacar aquellos que han centrado su análisis en la efectividad de los colores. Uno de ello es el estudio realizado por Donthu et al. (1993). Estos autores realizaron una investigación para comprobar el real alcance que tienen los colores utilizados en los avisos publicitarios sobre la recordación de la marca. Estos autores observaron, que los avisos que se basan en el blanco y el negro tienden a ser más recordados que aquellos que se basan en el color. Esto se debe a que los avisos en blanco y negro se destacan frente a la gran cantidad de avisos cuyos diseños tienen como fuente principal el color.

Otros estudios que se han realizado en esta línea han centrado su análisis en las características del texto expuesto en la publicidad. Este es el caso del estudio realizado por Hirschman y Wallendorf (1982). Estos autores, realizaron un experimento colocando dos tipos de avisos publicitarios. El primero de ellos estaba compuesto por un texto en el cual se incluía un máximo de 8 palabras y el segundo con un texto de 12 palabras, llegando con ello a la conclusión, que el público prefiere un texto corto y fácil de entender y que además esto facilita la recordación del aviso. A una conclusión similar llegaron con posterioridad los autores Donthu et al. (1993). De hecho, estos autores observaron que la publicidad exterior obtiene una mayor tasa de reconocimiento cuando se basa en un mensaje simple y breve. Incluso estos últimos autores señalan a través de su investigación, que los avisos con menos de siete palabras son más recordados que aquellos que contienen ocho o más palabras.

Otros estudios centraron sus análisis específicamente en el tema utilizado en los mensajes publicitarios. Uno de estos estudios es el realizado por Bhargava et al. (1994). Estos autores comprobaron que cuando el argumento del aviso se basa en un tema divertido o humorístico, el público le presta mayor atención. Esto se produce sobre todo, porque a las personas consciente o inconscientemente les agrada observar y vivir situaciones de este tipo.

Por ultimo, es necesario mencionar, aquellas investigaciones que han centrado sus estudios en la ubicación de los avisos. Estos estudios han sugerido, que desde la perspectiva de los conductores, los avisos ubicados a la derecha de la calle son más efectivos, y sobre todo cuando estos están ubicados en las carreteras y no en las ciudades, que son las que se caracterizan por tener una mayor cantidad de elementos distractores (Donthu et al., 1993). 


\section{Metodología}

Este estudio se realizó por medio de un cuestionario que se aplicó tanto en la vía pública como en el Metro de Santiago. En el caso del Metro las encuestas sólo pudieron realizarse fuera de los carros, ya que se prohíbe realizar esta tarea dentro de ellos. Las estaciones del Metro donde se realizaron las entrevistas fueron: Bellavista de La Florida, Universidad Católica y Estación Central. Las esquinas de la vía pública utilizadas par hacer las entrevistas fueron Vicuña Mackenna con Américo Vespucio, Alameda con Ahumada y Pedro de Valdivia con Providencia todas ellas arterias importantes de la ciudad de Santiago de Chile.

Para obtener la información necesaria para los análisis de la recordación y motivación de compra de la publicidad exterior, se realizaron diferentes tipos de preguntas. En el caso de la recordación se utilizó una pregunta abierta donde el encuestado debía señalar tres marcas, productos o servicios que recordaba haber visto publicitados en la vía pública o en el Metro, además de señalar la razón de su recordación. Estas respuestas abiertas se codificaron según la frecuencia de su incidencia, para así darle el mismo tratamiento que a las respuestas cerradas, es decir, tratarlas como variables nominales, y hacer el correspondiente análisis estadístico bajo esta restricción (moda, frecuencias y tablas de contingencia).

En cuanto a la motivación de compra, para su medición se utilizó una pregunta compuesta por cinco alternativas de respuesta (definitivamente lo motivan a comprar, probablemente lo motivan a comprar, no está seguro si lo motivan a comprar, probablemente no lo motivan a comprar y definitivamente no lo motivan a comprar). Este método de alternativas se presenta como la más idónea para realizar este tipo de estudios, dada la rapidez con la que se pueden obtener las respuestas y la facilidad de procesamiento de los datos.

Para el análisis de los datos se empleó el programa estadístico SPSS en su versión 11.0.

El cuestionario se aplicó a una muestra de 300 personas, por medio de un muestreo no probabilístico por cuotas según sexo, es decir, un número igual de entrevistas fueron aplicadas tanto a hombres como a mujeres.

El estudio se centró en edades que fluctúan entre los 21 y 61 años. Este grupo objetivo fue seleccionado por el hecho que ellos tienen poder adquisitivo al encontrarse en plena edad laboral.

En cuanto a la edad de las personas que participaron en el estudio, la mayoría de los encuestados se encontraban en el tramo entre los 21 y 30 años de edad, representando el $43,3 \%$ de la muestra, seguidos por aquellos que tenían entre 31 y 40 años y por aquellos que se encontraban entre los 41 y 50 años de edad, representando cada uno de estos tramos el $24 \%$ de la muestra. Finalmente, los individuos de menor representación resultaron ser aquellos que se encuentran con edades sobre los 51 años y sobre todo aquellos que tienen más de 61 años de edad, quienes en suma representan casi el $9 \%$ del total de los encuestados. 


\section{Análisis de la recordación}

Los resultados del estudio indicaron que la publicidad expuesta en la vía pública, cumple a lo menos con uno de sus objetivos básicos, que es llamar la atención a un número importante de transeúntes. De hecho, el 67\% de los encuestados recordaba avisos publicitarios expuestos en la vía pública o en el Metro (ver Tabla 1).

\subsection{Marcas más recordadas}

Considerando las marcas más recordadas por los entrevistados ${ }^{1}$, el estudio reveló que estas son Lider, con un $8,45 \%$ de la muestra, seguida por marcas como Ripley, Entel PCS, Jumbo, Teleserie de Canal 13, Actividades Culturales, Consalud y Falabella (ver Tabla 2).

Entre las marcas más recordadas, podemos destacar el caso de las teleseries de canal 13, que a pesar de tener poco tiempo de exposición en el mercado, son recordadas fundamentalmente, según los encuestados, por su cobertura (están presente en muchos lugares) y por las características gráficas de su publicidad (colores, diseño del aviso, entre otros). Por otro lado, es interesante destacar la recordación que obtuvo la marca JC Decaux (empresa que tiene la concesión publicitaria en el Metro de Santiago) la que sin tener como objetivo llegar al mercado compuesto por el público en general, sino más bien al de las marcas que pueden publicitar a través de esta empresa, ha logrado penetrar en la mente del consumidor. Este fenómeno, se podría explicar por dos razones. La primera de ellas, dice relación con las características de sus avisos ubicados en las dependencias del Metro. Son avisos con un formato simple y efectivo que utilizan en contraste el fondo azul oscuro y letras blancas, lo que según la literatura analizada produce una mayor recordación que aquellos que se basan en colores más fuertes (Donthu et al., 1993). La segunda razón tiene que ver con la amplia cobertura publicitaria en este medio. Estos avisos están presentes

\section{Tabla 1}

Recordación de Publicidad Exterior

\begin{tabular}{cccc}
\hline Respuesta & Frecuencia & Porcentaje del Total & Porcentaje Acumulado \\
\hline SI & 201 & 67 & 67 \\
NO & 99 & 33 & 100 \\
Total & 300 & 100 & \\
\hline
\end{tabular}

Fuente: Elaboración propia.

1 Es importante señalar que no se incluyeron la totalidad de las marcas recordadas, pues varias de ellas presentaban sólo una o muy pocas recordaciones, lo que resulta irrelevante para el análisis. 


\section{Tabla 2}

Recordación Total de Marca en Publicidad Exterior

\begin{tabular}{cccc}
\hline $\begin{array}{c}\text { Marcas o eventos } \\
\text { Recordados }\end{array}$ & Frecuencia & Porcentaje & $\begin{array}{c}\text { Porcentaje } \\
\text { de la Muestra }\end{array}$ \\
\hline Lider & 17 & 4,7 & 8,45 \\
Ripley & 14 & 3,8 & 6,96 \\
Entel PCS & 14 & 3,8 & 6,96 \\
Jumbo & 13 & 3,6 & 6,47 \\
Teleserie & 13 & 3,6 & 6,47 \\
Actividades Culturales & 13 & 3,6 & 6,47 \\
Consalud & 13 & 3,6 & 6,47 \\
Falabella & 13 & 3,6 & 6,47 \\
Adidas & 12 & 3,3 & 5,97 \\
JC Decaux & 11 & 3 & 5,47 \\
OMO & 10 & 2,7 & 4,98 \\
Sedal & 10 & 2,7 & 4,98 \\
Cine & 10 & 2,7 & 4,98 \\
LAN & 9 & 2,5 & 3,48 \\
VTR & 8 & 2,2 & 3,98 \\
Bomberos & 8 & 2,2 & 3,48 \\
Nike & 7 & 1,9 & 2,99 \\
Coca - Cola & 6 & 1,6 & 2,99 \\
Aviso Metro & 6 & 1,6 & 2,49 \\
Yo Tomo Leche & 5 & 1,4 & $100 \%$ \\
Total considerado & 212 & 58,1 & \\
\hline
\end{tabular}

Fuente: Elaboración propia.

en todas las estaciones del Metro, incluso con 2 ó 3 avisos por estación ${ }^{2}$, señal clara de que cobertura es importante para lograr una alta recordación de marca (Whitehill y Tinkham, 1990).

Por último, se puede destacar, el alto nivel de recordación que presentaron las "actividades culturales" que se patrocinan en el Metro de Santiago, comparado con aquellas marcas que invierten mayor cantidad de dinero en este recurso publicitario. Una de las razones que permiten explicar este fenómeno es el mayor interés que están mostrando las personas por las actividades culturales, según lo señalaron en sus respuestas los entrevistados. La otra razón, es el alcance que ha llegado a tener el Metro. De hecho, el Metro es un buen soporte para comunicar un mensaje publicitario a un gran número de personas. Cuando se comercializan productos de consumo masivo, el Metro

2 Esto ocurre porque muchos espacios publicitarios del Metro están vacíos, lo que ha obligado a JC Decaux a poner sus avisos invitando de esta manera a las empresas a invertir en estos espacios disponibles. 
se transforma en un excelente medio que permite un alto grado impacto y llegada a una audiencia masiva, ofreciendo además, claras posibilidades de "moldearse" a cada necesidad (Moles, 1976).

Lo anterior, se ve fuertemente respaldado al analizar la primera recordación espontánea de marcas publicitas en la vía pública o en el Metro, donde las actividades culturales se presentaron como las top of mind del consumidor (Tabla 3 ).

\subsection{Razones de la recordación}

La razón más recurrente que fue aludida por los encuestados, como la que permite recordar las marcas publicitadas en la vía pública o el Metro $^{3}$, fueron las promociones comunicadas por las marcas (ver Tabla 4).

Esto permite deducir, la sensibilidad que existe entre los consumidores a las promociones y los beneficios ofrecidos por las marcas. Lo que es consistente con los resultados observados por Bhargava et al. (1994). De hecho, cualquier cambio favorable en los beneficios ofrecidos, hace que el consumidor reaccione, recordando en consecuencia las marcas que hacen las promociones o que dicen tener los precios más bajos del mercado. Este es el caso de supermercados Líder, que como pudimos apreciar en el apartado anterior, es la marca más recordada (ver Tabla 2). Su estrategia competitiva (precios bajos siempre) coincide con la razón de su recordación, que se basa en las promociones de precio. Otras de las razones que se pueden desta- car, son el diseño y los colores del aviso y su cobertura geográfica.

Finalmente, se pueden destacar otros elementos que generalmente están presentes en la recordación de una marca, como son el atractivo del mensaje, su originalidad, el lugar donde se encuentra el aviso (disponibilidad) y la utilización de personajes conocidos en las campañas publicitarias. Con estos últimos se generan asociaciones positivas en el consumidor, pues se traspasan las características del personaje hacia la marca (Keller, 1993). Estos personajes generalmente son percibidos positivamente y con alto grado de credibilidad por la sociedad y son tomados como estereotipos o grupos de aspiración por parte del mercado objetivo.

\section{Análisis de la motivación de compra}

Para facilitar el análisis de la motivación de compra, se agruparon las cinco alternativas de respuesta que podían entregar los encuestados en sólo tres alternativas. Es así como las afirmaciones "definitivamente lo motivan a comprar" y "probablemente lo motivan a comprar", fueron interpretadas como una "motivación positiva de compra" hacia una marca recordada en la publicidad exterior. Por su parte las afirmaciones "probablemente no lo motivan a comprar" y "definitivamente no lo motivan a comprar" fueron interpretadas como una "motivación nega-

Para facilitar la tabulación y análisis de los resultados, se agruparon diversos conceptos en uno solo. 
Tabla 3

Primera recordación espontánea de marca en publicidad exterior

\begin{tabular}{|c|c|c|c|}
\hline Marca o actividad & Frecuencia & $\begin{array}{c}\text { Porcentaje } \\
\text { del Total }\end{array}$ & $\begin{array}{c}\text { Porcentaje } \\
\text { de la Muestra }\end{array}$ \\
\hline Actividades Culturales & 12 & 6,0 & 5,97 \\
\hline Sedal & 9 & 4,5 & 4,48 \\
\hline Lider & 9 & 4,5 & 4,48 \\
\hline $\mathrm{OMO}$ & 7 & 3,5 & 3,48 \\
\hline Jumbo & 7 & 3,5 & 3,48 \\
\hline Ripley & 6 & 3,0 & 2,99 \\
\hline Cine & 6 & 3,0 & 2,99 \\
\hline VTR & 6 & 3,0 & 2,99 \\
\hline Entel PCS & 6 & 3,0 & 2,99 \\
\hline Falabella & 6 & 3,0 & 2,99 \\
\hline LAN & 5 & 2,5 & 2,49 \\
\hline Teleserie & 5 & 2,5 & 2,49 \\
\hline Bomberos & 5 & 2,5 & 2,49 \\
\hline JC Decaux & 5 & 2,5 & 2,49 \\
\hline Adidas & 5 & 2,5 & 2,49 \\
\hline Intime & 4 & 2,0 & 1,99 \\
\hline París & 4 & 2,0 & 1,99 \\
\hline UNAB & 4 & 2,0 & 1,99 \\
\hline Coca-Cola & 4 & 2,0 & 1,99 \\
\hline Aviso Metro (huellas) & 4 & 2,0 & 1,99 \\
\hline Concurso Metro & 3 & 1,5 & 1,49 \\
\hline Nike & 3 & 1,5 & 1,49 \\
\hline $\mathrm{BCl}$ & 3 & 1,5 & 1,49 \\
\hline Nescafé & 2 & 1,0 & 1 \\
\hline Mc Donald's & 2 & 1,0 & 1 \\
\hline Pepsodent & 2 & 1,0 & 1 \\
\hline Bco. Santander Stgo. & 2 & 1,0 & 1 \\
\hline Axe & 2 & 1,0 & 1 \\
\hline TVN & 2 & 1,0 & 1 \\
\hline Intel & 2 & 1,0 & 1 \\
\hline Drive & 2 & 1,0 & 1 \\
\hline Fructis & 2 & 1,0 & 1 \\
\hline Yo Tomo & 2 & 1,0 & 1 \\
\hline Sprite Zero & 1 & 0,5 & 0,5 \\
\hline Bellsouth & 1 & 0,5 & 0,5 \\
\hline UDLA & 1 & 0,5 & 0,5 \\
\hline
\end{tabular}

Fuente: Elaboración propia. 


\section{Tabla 4}

Razones de la Recordación

\begin{tabular}{lcc}
\hline Razones & Frecuencia & Porcentaje del Total \\
\hline Promoción & 53 & 19,6 \\
Diseño y colores del aviso & 35 & 13 \\
Cobertura geográfica & 35 & 13 \\
Mensaje entregado & 19 & 07 \\
Personajes conocidos & 14 & 5,2 \\
Originalidad & 14 & 5,2 \\
Disponibilidad & 13 & 4,8 \\
Se relacionan con algo deportivo & 13 & 4,8 \\
Tamaño del aviso & 12 & 4,4 \\
Modelos que parecen & 11 & 4.1 \\
Imágenes mostradas & 11 & 4.1 \\
Llamativo del aviso & 9 & 3.3 \\
Comentarios de terceras personas & 9 & 3.3 \\
Luminosidad del aviso & 8 & 3.0 \\
El aviso entrega una advertencia & 4 & 1.5 \\
Es emotivo & 3 & 1.1 \\
La marca realiza eventos & 2 & 0.7 \\
Creatividad & 2 & 0.7 \\
Beneficios funcionales de la marca & 1 & 0.4 \\
Se relaciona con cultura & 1 & 0.4 \\
Muestra nuevas actividades & 1 & 0.4 \\
Total & 270 & 100 \\
\hline
\end{tabular}

Fuente: Elaboración propia.

tiva de compra". En cambio la afirmación "no está seguro si lo motivan a comprar" fue interpretada como una "motivación neutral de compra".

\subsection{Relación entre la motivación de compra y sexo}

Al analizar la tabla de contingencias que cruza las variables motivación de compra y sexo de los entrevistados (ver Tabla 5), se puede apreciar que de las 201 personas que recuerdan algún aviso publicitario en la vía pública o en el Metro, el 58,7\% manifiesta una motiva- ción positiva hacia la compra de las marcas publicitada, el $22,4 \%$ una motivación neutral y el 18,9\% una motivación negativa, sin registrarse diferencias apreciables entre hombres y mujeres.

Lo anterior se ve confirmado estadísticamente con la Prueba de Chi-Cuadrado que considera como hipótesis nula que no existe una relación entre motivación de compra y sexo (existe relación pvalue asociado $\mathrm{a}^{2}$ con un nivel de significancia menor a 0,05$)$. En este caso los resultados arrojaron un $p$-value $=0,916$. Esto implica que no se rechaza la hipótesis nula, y por lo tanto, podemos concluir 


\begin{tabular}{ccccc} 
& & \multicolumn{2}{c}{ Tabla 5 } \\
& Motivación de Compra según Sexo & \\
\hline \multirow{2}{*}{ MOTIVACIÓN } & & \multicolumn{2}{c}{ SEXo } & Total \\
\cline { 3 - 5 } & & Hombre & Mujer & \\
\hline \multirow{2}{*}{ Negativa } & Frecuencia & 21 & 17 & 38 \\
& Porcentaje & $20,0 \%$ & $17,7 \%$ & $18,9 \%$ \\
Neutral & Frecuencia & 23 & 22 & 45 \\
& Porcentaje & $21,9 \%$ & $22,9 \%$ & $22,4 \%$ \\
& Frecuencia & 61 & 57 & 118 \\
Positiva & Porcentaje & $58,1 \%$ & $59,4 \%$ & $58,7 \%$ \\
& Frecuencia & 105 & 96 & 201 \\
& Porcentaje & $100,0 \%$ & $100,0 \%$ & $100,0 \%$ \\
\hline
\end{tabular}

Fuente: Elaboración propia.

que no existe una relación estadísticamente significativa entre la motivación de compra generada por la publicidad exterior y el sexo de las personas entrevistadas (ver Tabla 6).

\subsection{Relación entre motivación de compra y edad}

En este apartado se analiza la relación entre la motivación de compra y la edad de las personas que recuerdan publicidad en la vía pública o en el Metro.

Considerando tres grupos de edades (entre 21 y 40 años; entre 41 y 50 años y los mayores de 51 años), podemos apreciar en la Tabla 7, que si bien se registra una motivación más positiva de compra en los grupos más jóvenes, no existe una relación clara entre la edad de las personas que recuerdan la publicidad exterior y su motivación por comprar las marcas publicitadas.
Este resultado se ve respaldado estadísticamente al realizar una prueba de Chi-Cuadrado. Con un p-value asociado $a^{2} 0.05$ (en este caso 0.41 ) no se rechaza la hipótesis nula, y por lo tanto se puede concluir que no existe una relación estadísticamente significativa entre la edad de las personas y la motivación de compra generada por la publicidad exterior (ver Tabla 8).

\section{Conclusiones}

Considerando que la muestra utilizada en este estudio es no probabilística, no es posible obtener resultados concluyentes, pero si es posible establecer hipótesis que puedan ser validadas posteriormente, a través de un estudio confirmatorio.

En primer lugar, podemos plantear que la publicidad exterior tiene un alto nivel de recordación, ya que dos tercios de la muestra recuerdan haber visto algún 


\section{Tabla 6}

\section{Prueba Chi-Cuadrado entre Motivación de Compra y Sexo}

\begin{tabular}{lccc}
\hline & Valor & g.l. & Sig. (Bilateral) \\
\hline Chi-Cuadrado de Pearson & $0,176^{*}$ & 2 & 0,916 \\
Razón de verosimilitud & 0,177 & 2 & 0,916 \\
Asociación Lineal por Lineal & 0,103 & 1 & 0,748 \\
№ de Casos Válidos & 201 & & \\
\hline
\end{tabular}

Nota: * 0 celdas $(0 \%)$ tienen frecuencias esperadas menores que 5 . La mínima frecuencia esperada es 18,15 . Fuente: Elaboración propia.

\section{Tabla 7}

Motivación de Compra según Edad

\begin{tabular}{|c|c|c|c|c|c|}
\hline \multirow[t]{2}{*}{ MOTIVACIÓN } & & \multicolumn{3}{|c|}{ EDAD } & \multirow[t]{2}{*}{ Total } \\
\hline & & 21 a 40 & 41 a 50 & 51 o más & \\
\hline \multirow[t]{2}{*}{ Negativa } & Frecuencia & 28 & 5 & 5 & 38 \\
\hline & Porcentaje & $18,7 \%$ & $13,9 \%$ & $33,3 \%$ & $18,9 \%$ \\
\hline \multirow[t]{2}{*}{ Neutral } & Frecuencia & 31 & 11 & 3 & 45 \\
\hline & Porcentaje & $20,7 \%$ & $30,6 \%$ & $20,0 \%$ & $22,4 \%$ \\
\hline \multirow[t]{3}{*}{ Positiva } & Frecuencia & 91 & 20 & 7 & 118 \\
\hline & Porcentaje & $60,7 \%$ & $55,6 \%$ & $46,7 \%$ & $58,7 \%$ \\
\hline & Frecuencia & 150 & 36 & 15 & 201 \\
\hline Total & Porcentaje & $100,0 \%$ & $100,0 \%$ & $100,0 \%$ & $100,0 \%$ \\
\hline
\end{tabular}

Fuente: Elaboración propia.

aviso publicitario en la vía pública o en el Metro.

Las marcas más recordadas a partir de la publicidad exterior corresponden a supermercados, multitiendas, empresas de telecomunicaciones, canales de televisión e instituciones de salud previsional.

Las razones más importantes de recordación, son las promociones, el diseño, la cobertura geográfica de los avi- sos, las características del mensaje entregado y la utilización de personajes conocidos. Como parte del diseño, se destacan el atractivo y la originalidad del mensaje, los colores y el tamaño del aviso publicitario.

Es necesario destacar que al parecer la publicidad exterior podría generar una alta motivación de compra en los consumidores, ya que el $58,7 \%$ de los entrevistados que recordaba haber visto al- 


\section{Tabla 8}

Prueba Chi-Cuadrado entre Motivación de Compra y Edad

\begin{tabular}{lccc}
\hline & Valor & g.l. & $\begin{array}{c}\text { Sig. Asintótica } \\
\text { (Bilateral) }\end{array}$ \\
\hline Chi-Cuadrado de Pearson & $3,974^{*}$ & 4 & 0410 \\
Razón de verosimilitud & 3,639 & 4 & 0457 \\
Asociación Lineal por Lineal & 1,145 & 1 & 0285 \\
№ de casos Válidos & 201 & & \\
\hline
\end{tabular}

Nota: * 2 celdas $(22,2 \%)$ tienen frecuencias esperadas menores que 5 . La mínima frecuencia esperada es $2,84$. Fuente: Elaboración propia.

gún aviso en la vía pública, dice tener una motivación positiva hacia la compra de las marcas publicitadas, el $22,4 \%$ manifiesta una motivación neutral y el 18,9\% una motivación negativa hacia la compra. Por último, no se registran diferencias estadísticamente significativas en la motivación de compra por sexo y por edad, lo que nos permitiría plantear la hipótesis que la publicidad exterior genera una alta motivación de compra en los consumidores, independiente de sus características demográficas.

Desde esta perspectiva, se deberían desarrollar futuras investigaciones sobre la publicidad exterior en Chile, con el objeto de validar las hipótesis planteada por la presente investigación exploratoria. Asimismo, se sugiere desarrollar futuras investigaciones comparativas sobre la efectividad de la publicidad exterior con la efectividad de la publicidad en medios publicitarios tradicionales como prensa, radio y televisión.

Finalmente, se sugiere más adelante desarrollar investigaciones sobre la efectividad de diferentes soportes publicitarios en vía pública, como son por ejemplo, el transporte urbano, paraderos de autobuses, edificios y letreros camineros.

Estas futuras investigaciones deberían orientarse a apoyar la planificación de medios de las empresas, con el fin de mejorar la asignación de recursos y así aumentar la eficiencia y la rentabilidad de la inversión publicitaria.

\section{Referencias Bibliográficas}

Bhargava, Mukesh y Donthu, Naveen (1993). "Sales Response to Outdoor Advertising". Canadian Journal of Marketing Research. 12, Canada, Marketing Research and Intelligence Association, pp. 87-97.

Bhargava, Mukesh; Donthu, Naveen y Caron, Rosanne (1994). "Improving the Effectiveness of Outdoor Advertising: Lessons from a Study of 282 Campaigns". Journal of Advertising Research. 34 (2), USA, American Marketing Association, pp. 46-55.

Donthu, Naveen; Cherian, Joseph y Bhargava, Mukesh (1993). "Factors Influencing Recall of Outdoor Advertising". Journal of Advertising Research. 33 (3), USA, American Marketing Association, pp. 64-72. 
Hewett, Wendell C. (1975). "The Significance of Human Curiosity in an Outdoor Advertising Experiment". Journal of Business. 48 (1), USA, University of Chicago Press, pp. 108-110.

Hirschman, Elizabeth y Wallendorf, Melanie (1982). "Motives Underlying Marketing Information Acquisition and Knowledge Transfer". Journal of Advertising. 11 (3), USA, American Academy of Advertising, pp. 25-31.

Keller, Kevin L. (1993). "Conceptualizing, Measuring, and Managing Customer-Based Brand Equity". Journal of Marketing. 57 (January), USA, American Marketing Association, pp. 01-22.

Moles, Abraham (1976). El Afiche en la Sociedad Urbana. Buenos Aires, Argentina, Editorial Piados.
Whitehill, Karen y Tinkham, Spencer (1990). "The Learning and Retention of Outdoor Advertising". Journal of Advertising Research. 33 (3), USA, American Marketing Association, pp. 47-51.

Woodside, Arch (1990). "Outdoor Advertising as Experiments". Journal of the Academy of Marketing Science. 18 (3), USA, Academy of Marketing Science, pp. 229-237.

Young, Elliot (1984). "Visibility Achieved By Outdoor Advertising". Journal of Advertising Research. 24 (4), USA, American Marketing Association, pp. 19-21. 